2 additional exams, $84(19.4 \%)$ had a negative result after the COVID-19 diagnosis, followed by a positive result. The proportion did not vary significantly across age groups (Fisher exact test, $P=.1821$ ) (Table 1 ). Among those 84 COVID-19 cases, median time from the negative swab and the following positive swab was 2 days. Only 2 persons had 1 positive result after 2 consecutive negative tests.

Negative RT-PCR tests followed, within few days, by a positive result among COVID-19 confirmed cases can be reasonably considered a false negative because the same patients had a positive test immediately afterward. Our population included both symptomatic and asymptomatic SARS-CoV-2 infections. Our results are important not only for hospitalized patients, who might be discharged based on false-negative results but also for asymptomatic cases who might break isolation based on tests that might by not reliable. If those persons are still infectious, they can spread the virus in the community.

Lippi et $\mathrm{al}^{6}$ described potential RT-PCR vulnerabilities that may affect the diagnostic accuracy of this technique, including both general preanalytical issues (collection, handling, transport and storage of the swabs, quality and volume of the collected material, interference from other substances) and analytical issues (choosing the right diagnostic window, validation of assays, harmonization, instrument functioning). Ways to minimize the risk of diagnostic errors include repeated collection of specimens in patients with suspicion of infection, training on swab collection, quality assurance for analytical procedures, and combination of clinical evidence with RT-PCR results. ${ }^{6}$ Laboratory parameters, such as lactate dehydrogenase, C-reactive protein, alanine aminotransferase, neutrophil count, ${ }^{7}$ and results of chest computed tomography ${ }^{8}$ can help define the disease stage.

We were able to assess only the proportion of false-negative tests among subjects with multiple swabs collected after a positive test. Conversely, if a subject had a negative test (either the first or another one) and no further swabs, it was not possible to assess whether the test was truly negative. Nonetheless, we assume that our results are generalizable to all tests. Thus, a first negative result should not be sufficient to neglect social distancing measures or use of personal protective equipment.

For a better understanding of the role and diagnostic accuracy of RT-PCR for SARS-CoV-2, further research should be conducted to assess viral load in respiratory specimens in patients with different severity of infection and at different time points.

Ethical considerations. All procedures contributing to this work comply with the Declaration of Helsinki. The analyses were based on anonymous administrative data, therefore patient consent and Ethical Committee approval were not required in Italy.

Acknowledgments. None.

Financial support. No financial support was provided relevant to this article.

Conflicts of interest. All authors report no conflicts of interest relevant to this article.

\section{References}

1. Covid-19. Situazione in Italia. Ministero della Salute website. http://www. salute.gov.it/portale/nuovocoronavirus/dettaglioContenutiNuovoCoronavirus. jsp?lingua $=i$ taliano $\& i d=5351 \&$ are $a=$ nuovoCoronavirus $\&$ menu $=$ vuoto. Accessed April 17, 2020.

2. Aggiornamento 16/04/2020 - ore 17.00. Ministero della Salute website. http://www.salute.gov.it/imgs/C_17_notizie_4505_0_file.pdf Accessed April 17, 2020.

3. Laboratory testing for coronavirus disease (COVID-19) in suspected human cases. World Health Organization website. https://www.who.int/publicationsdetail/laboratory-testing-for-2019-novel-coronavirus-in-suspected-humancases-20200117. Accessed March 25, 2020.

4. Li Y, Yao L, Li J, Chen L, Song Y, Cai Z, Yang C. Stability issues of RT-PCR testing of SARS-CoV-2 for hospitalized patients clinically diagnosed with COVID-19. J Med Virol 2020 Mar 26 [Epub ahead of print]. doi: 10.1002/ jmv.25786.

5. Xiao AT, Tong YX, Zhang S. False-negative of RT-PCR and prolonged nucleic acid conversion in COVID-19: rather than recurrence. J Med Virol 2020 Apr 9 [Epub ahead of print]. doi: 10.1002/jmv.25855.

6. Lippi G, Simundic AM, Plebani M. Potential preanalytical and analytical vulnerabilities in the laboratory diagnosis of coronavirus disease 2019 (COVID-19). Clin Chem Lab Med 2020 Mar 16 [Epub ahead of print]. doi: $10.1515 / \mathrm{cclm}-2020-0285$.

7. Mardani R, Ahmadi Vasmehjani A, Zali F, et al. Laboratory parameters in detection of COVID-19 patients with positive RT-PCR; a diagnostic accuracy study. Arch Acad Emerg Med 2020;8:e43.

8. Li D, Wang D, Dong J, et al. False-negative results of real-time reversetranscriptase polymerase chain reaction for severe acute respiratory syndrome coronavirus 2: role of deep-learning-based CT diagnosis and insights from two cases. Korean J Radiol 2020;21:505-508.

\title{
Severe complicated influenza declined during the prevention of COVID-19 in Taiwan
}

\author{
Chih-Jen Yang ${ }^{1,2}$ (1), Tun-Chieh Chen ${ }^{1,2}$, Shin-Huei Kuo ${ }^{1}$, Min-Han Hsieh ${ }^{1}$ and Yen-Hsu Chen ${ }^{1,2,3,4,5}$ \\ ${ }^{1}$ Department of Internal Medicine, Kaohsiung Municipal Ta-Tung Hospital, Kaohsiung Medical University, Kaohsiung, Taiwan, ${ }^{2} \mathrm{School}$ of Medicine, ${ }^{3} \mathrm{Graduate}$ \\ Institute of Medicine, ${ }^{4}$ Center of Tropical Medicine and Infectious Diseases, Kaohsiung Medical University, Kaohsiung, Taiwan and ${ }^{5}$ Department of Biological \\ Science and Technology, College of Biological Science and Technology, National Chiao Tung University, Hsinchu, Taiwan
}

\section{Dear Editor}

Corresponding Author: Yen-Hsu Chen, E-mail: infchen@gmail.com

Cite this article: Yang C-J, et al. (2021). Severe complicated influenza declined during the prevention of COVID-19 in Taiwan. Infection Control \& Hospital Epidemiology, 42: 499-501, https://doi.org/10.1017/ice.2020.272
The study of face masks use in Latin America reported by Soto et al is quite interesting ${ }^{1}$. SARS-CoV2 (COVID19) is a novel coronavirus that has caused a critical public threat worldwide. How to prevent the COVID-19 outbreak is a crucial issue and 


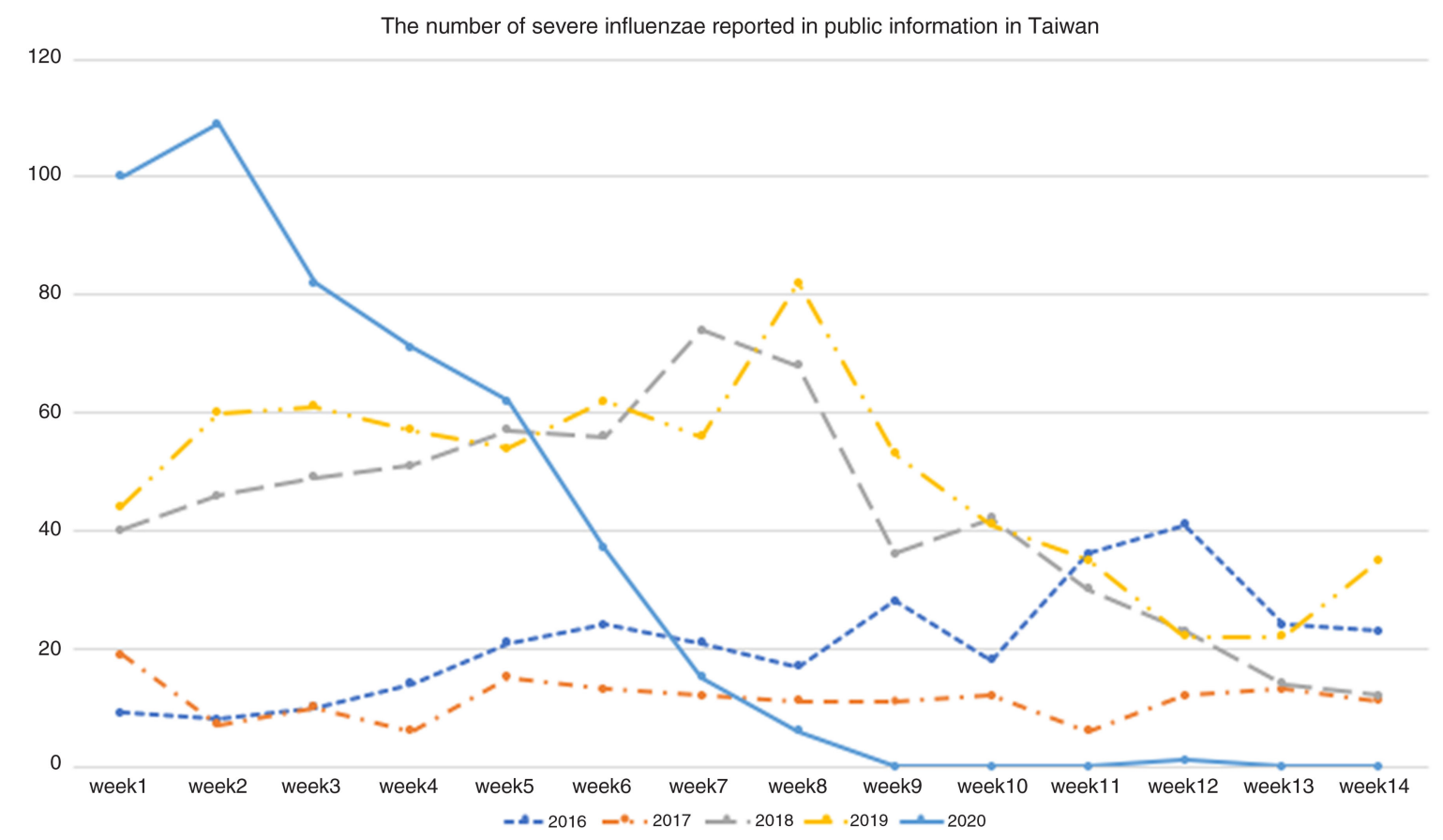

Fig. 1. The number of cases of reported severe complicated influenza from 2016 to 2020 in Taiwan according to the Taiwan CDC (https://www.cdc.gov.tw/).

community-based preparedness is important. SARS-CoV2 is transmitted through droplets and contact transmission routes, and the Taiwan government has taken many proactive and aggressive actions to prevent the transmission of COVID-19, including wearing face masks. These strategies have not only controlled COVID-19 transmission but also reduced influenza infection in Taiwan.

Although Taiwan is geographically close to China, the number of confirmed cases with COVID-19 in Taiwan is significantly lower than in other neighboring countries, with fewer than 450 confirmed COVID-19 cases and limited community transmission. The Taiwan government has acted swiftly since January to prevent the COVID-19 outbreak crisis by implementing measures such as border control, case identification and isolation, quarantine of suspected cases, extending holidays, travel restriction, education and big data integration. In addition, the export of face masks was prohibited on January 24, 2020 to avoid a shortage. In addition to these proactive early decisions, the Taiwan government acquired all available face masks and assigned most of them to hospital staff first in order to protect them from COVID-19. Moreover, mask making machine manufacturers were asked to step up face mask production capacity, which rose from 1.3 million per day to 13 million per day by the end of March 2020 .

Based on the previous experience of severe acute respiratory syndrome (SARS) in 2003, the Taiwan CDC encouraged residents to wear face masks and perform hand hygiene, especially hospital staff and people in crowded places when the COVID-19 outbreak was initially reported in Wuhan, China in January $2020^{2}$. Recently, the Taiwan CDC announced regulations requiring all passengers using public transportation to wear face masks all the time and follow the social distancing recommendations.

This successful policy not only controlled the community transmission of COVID-19, but also had an unexpected effect on lowering the incidence of severe complicated influenza after the sixth week this year based on the public records of the Taiwan CDC (Fig. 1). In Taiwan, the main influenza period occurs in the winter, from late November through March. Influenza cases are not required to be reported. However, patients with serious influenza complications who need intensive care unit treatment and associated deaths are classified as having a notifiable disease, "severe complicated influenza", and these cases have to be reported within one week. Hundreds of people die of severe influenza in Taiwan every year. Since January 2020, the Taiwan CDC has required that all patients with suspected COVID-19 should also be checked for both SARS-CoV-2 and influenza virus infections. According to the Taiwan CDC, the number of cases of severe complicated influenza decreased markedly since the sixth week this year. Moreover, only one severe complicated influenza case was reported in March, compared to approximately 10 to 50 cases per month from January to March reported in the past five years. Hand hygiene and social distancing are both recommended strategies to prevent influenza based on systematic reviews ${ }^{3,4}$, and based on the Taiwan CDC data, we believe that a face mask wearing policy also plays an important role in the prevention influenza infection.

Face masks, when fitted properly, effectively disrupt the forward momentum of particles expelled from a cough or sneeze, preventing respiratory disease transmission ${ }^{5}$. In addition, a previous case report also showed that wearing a face mask helped to reduce the transmission of influenza during the COVID-19 pandemic ${ }^{6}$. Face masks appear to be highly efficient at filtering pathogens, including influenza and rhinoviruses ${ }^{7}$, and their use should be encouraged during influenza pandemics ${ }^{3,8}$. For healthcare workers, a meta-analysis of randomized controlled trials indicated a protective effect of masks and respirators against clinical respiratory illnesses (risk ratio [RR]: 0.59; 95\% confidence interval [CI]: 0.46-0.77) and influenza-like illness (RR: 0.34; 95\% CI: 0.14-0.82). Furthermore, even pooled analysis of 
observational studies revealed the benefit of wearing masks (odds ratio $[\mathrm{OR}]=0.13 ; 95 \% \mathrm{CI}: 0.03-0.62)$ and respirators $(\mathrm{OR}=0.12$; 95\% CI: 0.06-0.26) against SARS.

Although Some studies have been against the use of face masks during the COVID-19 pandemic $^{9}$ and one meta-analysis reported that the use of face masks to prevent transmission of influenza virus is still controversial ${ }^{10}$, the mask-wearing policy accompanied with hand hygiene and social distancing appear to have prevented both severe complicated influenza and COVID-19 in Taiwan. In April 2020, the Taiwan Government donated approximately 10 million face masks to countries hit hardest by SARS-COV-2, including the United States and the European Union. We hope that our experience can help other regions to overcome the COVID-19 pandemic as quickly as possible.

Acknowledgement. We thank Professor Ho Pei-Shan, Division of Medical Statistics and Bioinformatics, Department of Medical Research, Kaohsiung Medical University, Kaohsiung, Taiwan.

Contribution. CJ Yang and YH Chen, study design and draft the manuscript. TC Chen, SH Kuo, MH Hsieh, data collection, analysis and discussion.

All authors approved the final manuscript.

Funding. No funding.

Financial support. None.

Conflict of interests. No conflict of interest to be declared.

\section{REFERENCES}

1. Soto DM, Cardona Maya WD, Londono E, Bueno Sanchez JC. The feasibility of generalized face mask usage during COVID-19 pandemic: a perspective from Latin America. Infect Control Hosp Epidemiol 2020:1-4. doi: 10.1017/ ice.2020.227.

2. Yang W, Cao Q, Qin L, Wang X, Cheng Z, Pan A, Dai J, Sun Q, Zhao F, $\mathrm{Qu}$ J, Yan F. Clinical characteristics and imaging manifestations of the 2019 novel coronavirus disease (COVID-19): A multi-center study in Wenzhou city, Zhejiang, China. J Infect 2020;80:388-393. doi: 10.1016/ j.jinf.2020.02.016.

3. Qualls N, Levitt A, Kanade N, Wright-Jegede N, Dopson S, Biggerstaff M, Reed C, Uzicanin A, Group CDCCMGW. Community Mitigation Guidelines to Prevent Pandemic Influenza - United States, 2017. MMWR Recomm Rep 2017;66:1-34. doi: 10.15585/mmwr.rr6601a1.

4. Wong VW, Cowling BJ, Aiello AE. Hand hygiene and risk of influenza virus infections in the community: a systematic review and meta-analysis. Epidemiol Infect 2014;142:922-932. doi: 10.1017/S095026881400003X.

5. Sim SW, Moey KS, Tan NC. The use of facemasks to prevent respiratory infection: a literature review in the context of the Health Belief Model. Singapore Med J 2014;55:160-167. doi: 10.11622/smedj.2014037.

6. Liu X, Zhang S. COVID-19 : Face Masks and Human-to-human Transmission. Influenza Other Respir Viruses 2020 doi: 10.1111/irv.12740.

7. Zhou SS, Lukula S, Chiossone C, Nims RW, Suchmann DB, Ijaz MK. Assessment of a respiratory face mask for capturing air pollutants and pathogens including human influenza and rhinoviruses. J Thorac Dis 2018;10:2059-2069. doi: 10.21037/jtd.2018.03.103.

8. Zhang CQ, Chung PK, Liu JD, Chan DKC, Hagger MS, Hamilton K. Health Beliefs of Wearing Facemasks for Influenza A/H1N1 Prevention: A Qualitative Investigation of Hong Kong Older Adults. Asia Pac J Public Health 2019;31: 246-256. doi: 10.1177/1010539519844082.

9. Bae S, Kim MC, Kim JY, Cha HH, Lim JS, Jung J, Kim MJ, Oh DK, Lee MK, Choi SH, Sung M, Hong SB, Chung JW, Kim SH. Effectiveness of Surgical and Cotton Masks in Blocking SARS-CoV-2: A Controlled Comparison in 4 Patients. Ann Intern Med 2020 doi: 10.7326/M20-1342.

10. Cowling BJ, Zhou Y, Ip DK, Leung GM, Aiello AE. Face masks to prevent transmission of influenza virus: a systematic review. Epidemiol Infect 2010;138:449-456. doi: 10.1017/S0950268809991658.

\title{
Influence of different definitions of central venous catheter-related bloodstream infections on epidemiological parameters in cancer patients
}

\author{
Enrico Schalk $M D^{1}$ (1), Maria J.G.T. Vehreschild $M^{2,3,4}$ and Lena M. Biehl MD ${ }^{2,3}$ \\ ${ }^{1}$ Department of Hematology and Oncology, Otto-von-Guericke University Magdeburg, Medical Center, Magdeburg, Germany, ${ }^{2}$ Department I of Internal Medicine, \\ Faculty of Medicine and University Hospital of Cologne, University of Cologne, Cologne, Germany, ${ }^{3}$ German Center for Infection Research (DZIF), Site Bonn/ \\ Cologne, Cologne, Germany and ${ }^{4}$ Department of Internal Medicine, Infectious Diseases, Goethe University Frankfurt, Frankfurt am Main, Germany
}

To the Editor-Especially for cancer patients, there are multiple coexisting definitions for central venous catheter (CVC)-related bloodstream infections (CRBSIs). Therefore, it is difficult to make comparisons across studies. Furthermore, a considerable number of publications (39 of 190, 21\%) did not report the CRBSI definition or cite a reference for the used definition. ${ }^{1}$ To complicate matters further, guidelines on diagnosis of CRBSIs are subject to change over time. ${ }^{2,3}$ For example, in 2003, the Infectious Diseases Working Party of the German Society of Hematology

\footnotetext{
Author for correspondence: Enrico Schalk, E-mail: enrico.schalk@med.ovgu.de
}

Cite this article: Schalk E, Vehreschild MJGT, and Biehl LM. (2021). Influence of different definitions of central venous catheter-related bloodstream infections on epidemiological parameters in cancer patients. Infection Control \& Hospital Epidemiology, 42: 501-503, https://doi.org/10.1017/ice.2020.274 and Medical Oncology (AGIHO) proposed to distinguish definite (dCRBSIs), probable (pCRBSIs), and possible (possCRBSIs) CRBSIs. ${ }^{4}$ Although these terms are still part of their current guideline, the exact definitions have been adjusted slightly over the years. ${ }^{5,6}$ In addition, dCRBSIs and pCRBSIs are often combined (dpCRBSIs) for reporting purposes. ${ }^{7,8}$

Recently, we provided comparative epidemiological data on CRBSIs from a randomized controlled trial (RCT) and a registry study in high-risk patients with hematological malignancies. ${ }^{7}$ In the RCT data set, the 2008 AGIHO definitions for CRBSIs were initially used, ${ }^{5}$ whereas the registry applied the 2012 AGIHO definitions. $^{6}$ The 2 guidelines differ in their definition of pCRBSIs and possCRBSIs. In brief, criteria for pCRBSIs and possCRBSIs are more strict in the newer guideline (see Supplementary Table S1 for a 